\title{
Erratum to: Abstract supplement to the 8th World Congress on Trauma, Shock, Inflammation and Sepsis - TSIS 2010
}

\author{
The Publisher
}

Published online: 3 June 2010

(C) Springer Basel AG 2010

\section{Erratum to: Inflamm Res (2010) 59 (Suppl 1):S9-S166 DOI 10.1007/s00011-010-0170-z}

Unfortunately the following abstracts contained mistakes.

A 22 The content of the abstract and the corresponding authors are corrected as follows:

\section{Systemic delivery of recombinant angiopoietin-1 ameliorates multiple-organ dysfunction syndrome in experimental abdominal sepsis}

Philipp Kuempers, Alexander-Henrik Lukasz, Joon-Keun Park, Matijs van Meurs, Christian Koenecke, Sascha David

Objective: Endothelial activation leading to vascular barrier breakdown plays an essential role in the pathophysiology of multiple-organ dysfunction syndrome (MODS) in sepsis. Binding of the vesselderived growth factor Angiopoietin-1 (Ang-1) to its endothelialspecific Tie2 receptor protects the vasculature from leakage and maintains vessel integrity. We examined the potential therapeutic benefit of systemically delivered recombinant Angiopoietin-1 in mice with septic MODS.

Methods: Using the caecal ligation and puncture (CLP) model, 8-10 week-old 129SV mice were treated with either Ang-1 (500 ng i.v.) or placebo (phosphate-buffered saline) immediately before CLP and every $8 \mathrm{~h}$ thereafter. Sham-operated animals served as timematched internal controls.

Results: Ang-1 treatment significantly reduced sepsis-induced vascular leakage while preventing leukocyte diapedesis and infiltration in the lung. Moreover, systemic delivery of Ang-1 mitigated renal intercellular adhesion molecule-1 and vascular cell adhesion molecule-1 protein expression, the number of Gr1-positive leucocytes that

The online version of the original article can be found under doi: 10.1007/s00011-010-0170-z.

The Publisher

Springer Basel AG, Basel, Switzerland infiltrated the kidney, and prevented increases in serum creatinine and urea levels. However, loss of Tie 2 mRNA and protein from the renal microvasculature was not rescued by Ang-1 treatment. Finally, increased resistance to MODS was reflected by an improved KaplanMeier survival rate in Ang-1-treated mice.

Conclusions: Our study demonstrates that administration of exogenous recombinant Ang-1 ameliorates MODS and improves survival time in experimental abdominal sepsis, probably via preventing excessive vascular inflammation and leakage.

Corresponding Author: Philipp Kuempers, Medizinische Hochschule Hannover, Department of Nephrology, Carl-Neuberg-Str. 1, 30625 Hannover, Germany, pkuempers@gmx.de

A 165 The authors are corrected as follows:

Differential stimulation of trauma related toll-like receptors from peripheral blood mononuclear cells in children compared to adults

James H. Wood, David A. Partrick, Ernest E. Moore, Ronald J. Harbeck, Richard B. Johnston Jr.

Objective: Toll-like receptors (TLRs) play a key role in innate immunity, and specific members of the TLR family (TLR $1 / 2,2 / 6,4$, and 9) contribute to trauma-induced inflammation, which can result in multiple organ failure (MOF). Post-injury MOF occurs less frequently in children than in adults, and we therefore hypothesize that peripheral blood mononuclear cells (PBMCs) from children will exhibit a blunted pro-inflammatory cytokine response to in-vitro stimulation with trauma-TLR specific ligands.

Methods: PBMCs isolated from blood samples of 20 healthy children (age range 2-12 years) and 17 healthy adults (age range 2466 years) were suspended in RPMI and plated in 96-well culture dishes at a concentration of $2 \times 10^{5}$ cells/well. Cultured cells were stimulated with TLR-specific ligands (Pam3CSK4: TLR 1/2; Zymosan: TLR 2/6; LPS: TLR 4; and ODN 2216: TLR 9) for $24 \mathrm{~h}$. TNF-a levels in supernatants were measured by ELISA. Data are mean \pm SEM.

Results: Stimulation of TLR $1 / 2$ resulted in higher levels of TNF-a production in adults compared to children (498 \pm 106 vs. $280 \pm 48 \mathrm{pg} / \mathrm{ml}$ ), although the difference did not reach statistical significance $(P=0.06)$ due to wide individual variation. TNF-a 
production in response to other ligands was similar between children and adults.

Conclusions: Age-related differences in TNF-a production resulting from TLR $1 / 2$ stimulation in PBMCs could contribute to the lower incidence of MOF in injured children. However, further work is needed to more clearly define the role of TLR $1 / 2$ in injury related inflammation and post-injury MOF. TNF-a production in response to stimulation of other trauma-associated TLRs is likely not implicated in age-related discrepancies in the incidence of postinjury postinjury MOF.

Table

\begin{tabular}{lrrr}
\hline & \multicolumn{1}{c}{ Children } & \multicolumn{1}{l}{ Adults } & $P$ value \\
\hline TLR 1/2 & $280 \pm 48$ & $498 \pm 106$ & 0.06 \\
TLR 2/6 & $1,330 \pm 169$ & $1,305 \pm 134$ & 0.91 \\
TLR 4 & $1,114 \pm 165$ & $1,336 \pm 172$ & 0.35 \\
TLR 9 & $984 \pm 148$ & $1,146 \pm 235$ & 0.62 \\
\hline
\end{tabular}

PBMC TNF-a production in response to TLR-specific ligands. Mean $\pm \mathrm{SEM}(\mathrm{pg} / \mathrm{ml})$

Corresponding Author: David Partrick, MD, The Children's Hospital, University of Colorado, Department of Pediatric Surgery, 13123 East 16th Avenue, B-323, Aurora, CO 80045, USA, partrick.david@tchden.org

A 390 The authors and the corresponding author's mail address are corrected as follows:

\section{A potential new serum marker for concussion injury in professional ice hockey sport: Pilot Study on S-100}

Karin Kiechle, Kai Fehske, Gerrit Oedekoven, Viktoria Bogner, Wolf Mutschler, Peter Biberthaler

Objective: Minor traumatic brain injury is a relevant problem in contact sports, such as American football, ice hockey and boxing. In this context, concussion injury has been identified as one of the major reasons for posttraumatic sequela. Hence, professional sport team physicians take out professional players after concussion injury, which implicates substantial sportive and financial problems for the team. So far, no quantitative and rapid available parameter is available to identify those individuals at risk. In this respect, the measurement of S-100 has been identified recently for identification of patients suffering from intracranial bleeding after TBI. So far, it remains unclear if measurement of S-100 might allow for identification of concussion injuries in contact sport. Hence, the aim of this study was, to measure S-100 after concussion injuries in ice hockey players and compare the results to standard clinical tests.

Patients and methods: Into this study, we included all professional players $(n=23)$ of a national leage team in Germany and draw samples during non-training summer period as negative intra-individual control and after a traumatic event. We calculated the ratio between the serum concentration of S-100 (Elecsys S100, Roche Diagnostics $\mathrm{GmbH}$ ) after a substantial concussion injury and the individual negative control concentration during training pause and after sharp physical activity. Furthermore, concussion victims were analyzed using clinical SCAT neurological examination form. All data is given as mean $\pm \mathrm{SD}, \mathrm{S}-100$ values are given in $[\mu \mathrm{g} / \mathrm{L}]$.

Results: During the winter season 2008/2009 4 concussion events and complete blood samples as well as SCAT data were recorded. All players had positive result in SCAT testing and stopped playing. The S-100 control values without training were $0.1 \pm 0.02$, after training $0.1 \pm 0.4$ and after concussion $0.2 \pm 0.08$. The individual slope between negative control and training was $1.3 \pm 0.52$, between concussion and training $2.3 \pm 0.83$ and between concussion and untrained [SG1] control $2.7 \pm 0.62$, respectively.

Conclusions: Although still preliminary, our data suggest for the first time that measurement of S-100 might allow for valid quantitative results for identification of individuals suffering a substantial concussion injury while performing contact sport. Hence, we recommend that further studies on extended collectives are performed to secure the potential diagnostic value of S-100 measurement in contact sports. Corresponding Author: Peter Biberthaler, MD, Ludwig-MaximiliansUniversity of Munich, Campus Innenstadt, Department of Trauma and Ortho, Nussbaumstr.20, 80336 Munich, Germany, peter.biberthaler@ med.uni-muenchen.de 